\title{
Descompressão lombar versus fusão espinhal em ambiente ambulatorial privado: Um estudo retrospectivo com três anos de acompanhamento*
}

\section{Lumbar Decompression Versus Spinal Fusion in a Private Outpatient Setting: A Retrospective Study with Three Years of Follow-up}

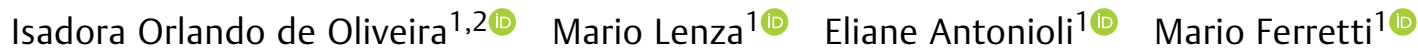

${ }^{1}$ Departamento de Ortopedia e Traumatologia, Hospital Israelita

Endereço para correspondência Mario Ferretti, PhD, Departamento Albert Einstein, São Paulo, SP, Brasil

2 Instituto Wilson Mello, Campinas, SP, Brasil de Ortopedia e Traumatologia, Hospital Israelita Albert Einstein, Avenida Albert Einstein 627/701, bloco A1, Jardim Leonor, São Paulo, Rev Bras Ortop 2021;56(6):766-771. SP, 05652-900, Brasil (e-mail: marioferretti@gmail.com).

\author{
Resumo \\ Palavras-chave \\ - avaliação de \\ resultados em \\ cuidados de saúde \\ - doenças \\ musculoesqueléticas \\ - dor nas costas \\ - coluna vertebral \\ - intervenções \\ cirúrgicas
}

Objetivo Comparar a dor, a função, a qualidade de vida e os eventos adversos da descompressão lombar e da fusão espinhal em pacientes com patologias degenerativas da coluna vertebral que participaram de um programa de segunda opinião para cirurgias de coluna com acompanhamento de 36 meses.

Métodos Os dados desta coorte retrospectiva foram obtidos de um sistema de saúde privado entre junho de 2011 e janeiro de 2014. A amostra do estudo foi composta por 71 pacientes encaminhados para cirurgia de coluna lombar. Os desfechos para comparações entre a descompressão lombar e a fusão espinhal foram qualidade de vida (avaliada pelo questionário EuroQoL 5D), dor (medida pela Escala Numérica de Classificação de Dor) e função (avaliada pelo Questionário de Incapacidade de Roland Morris) no início do estudo e aos 12 e 36 meses de acompanhamento pós-cirúrgico. As definições de recuperação foram estabelecidas pela diferença mínima clinicamente importante (DMCl). As diferenças basais entre os grupos foram analisadas por teste $t$ não pareado, e as diferenças nas pontuações dos instrumentos entre os momentos, por modelos mistos generalizados. Os resultados foram apresentados como valores médios ajustados pelos modelos e intervalos de confiança de $95 \%$.

Resultados No total, 22 pacientes foram submetidos à artrodese, e 49 pacientes, à descompressão lombar. Quanto às comparações de achados antes e depois das

\footnotetext{
Estudo desenvolvido no Departamento de Ortopedia e Traumato-

logia, Hospital Israelita Albert Einstein, São Paulo, SP, Brasil.
}

recebido

24 de Julho de 2020

aceito

17 de Setembro de 2020

Publicado on-line

Setembro 11, 2021
DOI https://doi.org/

10.1055/s-0041-1724083.

ISSN 0102-3616.
(C) 2021 . Sociedade Brasileira de Ortopedia e Traumatologia. All
rights reserved.
This is an open access article published by Thieme under the terms of the
Creative Commons Attribution-NonDerivative-NonCommercial-License,
permitting copying and reproduction so long as the original work is given
appropriate credit. Contents may not be used for commercial purposes, or
adapted, remixed, transformed or built upon. (https://creativecommons.org/
licenses/by-nc-nd/4.0/)
Thieme Revinter Publicações Ltda., Rua do Matoso 170, Rio de
Janeiro, RJ, CEP 20270-135, Brazil 


\begin{abstract}
Keywords

- outcome assessment, health care

- musculoskeletal diseases

- back pain

- spine

- surgical interventions

Objective To compare pain, function, quality of life and adverse events of lumbar decompression and spinal fusion in patients with degenerative spinal pathologies who participated in a second opinion program for spinal surgeries with a 36-month follow-up. Methods The data for this retrospective cohort were withdrawn from a private healthcare system between June 2011 and January 2014. The study sample consisted of 71 patients with a lumbar spine surgical referral. The outcomes for the comparisons between lumbar decompression and spinal fusion were quality of life (evaluated through the EuroQoL 5D), pain (measured by the Numerical Rating Scale) and function (assessed through the Roland Morris Disability Questionnaire) measured at baseline, and at 12 and 36 months after the surgical procedures. The definitions of recovery were established by the minimal clinically important difference (MCID). The baseline differences between the groups were analyzed by non-paired $t$-test, and the differences in instrument scores between time points, by generalized mixed models. The results were presented as mean values adjusted by the models and $95 \%$ confidence intervals. Results Concerning the surgical techniques, 22 patients were submitted to spinal fusion and 49 patients, to lumbar decompression. As for the comparisons of the findings before and after the surgical interventions, the MCID was achieved in all outcomes regarding quality of life, pain and function at both time points when compared to baseline scores Moreover, concerning the complication rates, only lumbar decompression presented a surgical rate of $4 \%(n=3)$ for recurrence of lumbar disc hernia.

Conclusion Patients with degenerative spinal pathologies present improvements in longterm outcomes of pain, function and quality of life which are clinically significant, no matter the surgical intervention.
\end{abstract}

intervenções cirúrgicas, a DMCI foi alcançada em todos os desfechos de qualidade de vida, dor e função nos dois pontos de acompanhamento em relação aos escores basais Em relação às complicações, apenas a descompressão lombar apresentou $4 \%(n=3)$ de taxa cirúrgica de recidiva da hérnia de disco lombar.

Conclusão Pacientes com patologias espinhais degenerativas apresentam melhoras nos desfechos de dor, função e qualidade de vida em longo prazo que são clinicamente significativas e independentes da intervenção cirúrgica.

\section{Introdução}

A doença articular degenerativa é a principal causa de deficiência crônica em todo o mundo, e é geralmente associada a dor, bloqueio articular, e rigidez. ${ }^{1} \mathrm{O}$ atual aumento da realização de cirurgias de coluna está relacionado aos processos degenerativos da coluna lombar; nos últimos anos, a incidência de procedimentos lombares cresceu dez vezes mais em comparação às demais cirurgias ortopédicas, como artroplastias totais de quadril ou joelho. ${ }^{2}$

Uma consequência da degeneração espinhal foi descrita pela primeira vez por Verbiest em 1954, e é definida pelo estreitamento do canal espinhal e consequente compressão da medula espinhal. ${ }^{3}$ Segundo o autor ${ }^{4}$, os sintomas de compressão da raiz nervosa por hipertrofia dos processos articulares ocorriam com o paciente em posição ortostática, principalmente durante a marcha. Outros estudos relataram que a compressão das estruturas nervosas se deve a outros componentes, como hipertrofia do ligamento amarelo (ligamentum flavum), cistos sinoviais adjacentes à faceta, e perda de altura do disco intervertebral. ${ }^{5}$
A princípio, os pacientes sintomáticos devem ser submetidos ao tratamento conservador com fisioterapia e medicamentos. Durante a avaliação, porém, uma pequena proporção de patologias espinhais graves ou com comprometimento da raiz nervosa é detectada, e o paciente é encaminhado para cirurgia. ${ }^{6}$ 0 programa de segunda opinião foi proposto para analisar os riscos e custos de procedimentos cirúrgicos desnecessários, e promove uma prática mais ética e benéfica para os pacientes e um serviço padrão para o tratamento da dor lombar. ${ }^{7}$

A descompressão cirúrgica está indicada em caso de insucesso do tratamento conservador, de desenvolvimento de síndrome da cauda equina, ou de déficit motor progressivo. ${ }^{8}$ Por outro lado, a fusão lombar é indicada em pacientes com instabilidade da coluna lombar. Este procedimento pode ser realizado por meio de fusão posterolateral (PL), com colocação de enxerto ósseo entre os processos transversos, ou técnica de fusão intersomática (IS), em que o enxerto ósseo é posicionado entre os corpos vertebrais.

A fusão IS é indicada quando a fonte da dor é o disco intervertebral. Independentemente de seu uso generalizado 
atual, poucas evidências comprovam sua superioridade. ${ }^{9}$ Apesar de tecnicamente mais difícil, a fusão IS dá melhor apoio para a coluna anterior, permite a descompressão foraminal indireta, restaura a lordose, e facilita a remoção do disco intervertebral, um importante fator de dor. ${ }^{10}$ A fusão PL é indicada quando a fonte de dor é a articulação facetária. ${ }^{11}$ Esta técnica é de execução mais fácil e tem menor índice de complicações, mas pode aumentar a dor devido à necessidade de maior exposição e danos aos músculos paravertebrais. ${ }^{12}$ Em relação aos desfechos, como dor e disfunção, a literatura é inconclusiva, pois não se determinou se uma abordagem oferece certo ganho em comparação com a outra. ${ }^{13,14}$

Apesar desses dados, discute-se qual é a intervenção mais adequada: a a fusão isolada ou a descompressão. ${ }^{11,15}$ Portanto, o objetivo deste estudo é comparar a dor, a função, e a qualidade de vida de pacientes com patologias degenerativas da coluna vertebral e submetidos à descompressão lombar ou fusão espinhal que participaram de um programa de segunda opinião para cirurgias de coluna com acompanhamento de 36 meses.

\section{Métodos}

\section{Delineamento Experimental}

Os dados para esta coorte retrospectiva foram retirados de um programa de segunda opinião para cirurgias de coluna conduzido em um sistema de saúde privado. Este sistema inclui pacientes com planos de saúde que receberam uma indicação para cirurgia por um profissional especializado em coluna vertebral do setor privado, e cujas seguradoras de saúde no Brasil ofereceram uma segunda opinião.

Os pacientes foram avaliados quanto à elegibilidade entre junho de 2011 e janeiro de 2014, e acompanhados por 36 meses. Os critérios de elegibilidade foram: ter indicação para cirurgia da coluna devido a doenças degenerativas da coluna lombar, como doença do disco intervertebral, espondilolistese degenerativa, estenose do canal lombar, dor lombar facetária e instabilidade lombar; não ter contraindicação para anestesia geral; ser capaz de compreender a língua portuguesa; e concordar em participar do estudo. Pacientes com fraturas da coluna vertebral, escoliose superior a 20 graus, deformidades congênitas, tumores espinhais, gravidez confirmada ou suspeita, e que não puderam completar o acompanhamento foram excluídos do estudo.

\section{Intervenções Cirúrgicas}

Os procedimentos cirúrgicos foram realizados ou supervisionados por 14 cirurgiões seniores (neurocirurgiões ou cirurgiões ortopédicos) com mais de 15 anos de experiência. Todos os pacientes foram submetidos à descompressão ou fusão. As decisões relativas ao procedimento realizado e aos níveis abordados foram baseadas nas diretrizes da prática clínica, considerando os achados ao exame clínico, os critérios de instabilidade, e a estenose espinhal.

\section{Coleta de Dados e Acompanhamento}

Os dados basais, como características sociodemográficas, estado geral de saúde e qualquer doença associada, foram coletados no hospital logo antes do procedimento cirúrgico por um assistente de pesquisa (cego quanto ao objetivo do estudo), assim como todas as medidas de desfechos. Os dados de acompanhamento em longo prazo foram coletados por telefone 12 e 36 meses após a cirurgia. Os dados foram verificados por dois coordenadores do estudo, e, em caso de ausência de dados, os bancos de dados foram cruzados para a recuperação das informações.

\section{Desfechos}

Os desfechos deste estudo foram dor, função e qualidade de vida, avaliados por questionários específicos, no início do estudo e aos 12 e 36 meses de acompanhamento após a cirurgia. Todos os instrumentos foram traduzidos e submetidos à adaptação transcultural para o português brasileiro e à análise de suas propriedades de mensuração. ${ }^{16-18}$ Além disso, as taxas de complicações e recidivas foram avaliadas pelos coordenadores do estudo com base no banco de dados do hospital e no prontuário dos pacientes.

Pedimos para os participantes classificarem sua dor média na última semana conforme a Escala Numérica (EN) de Classificação da Dor, de 11 pontos, que varia de 0 a 10 (em que 0 representa "sem dor", e 10 representa "a pior dor que o paciente poderia sentir"). ${ }^{19}$

Além disso, a função relacionada à dor nas costas foi autoavaliada pelo Questionário de Incapacidade de Roland Morris (QIRM), em que cada pergunta vale um ponto, e os escores variam de 0 (sem incapacidade) a 24 (incapacidade grave). ${ }^{18}$

A qualidade de vida relacionada à saúde foi medida pelo EuroQoL (EQ-5D), um instrumento de avaliação que utiliza cinco dimensões (5D: mobilidade, autocuidado, atividades habituais, dor/desconforto, e ansiedade/depressão) para gerar uma pontuação de 0 a 1 (pior a melhor). ${ }^{17}$

A diferença mínima clinicamente importante (DMCI), que atribui um valor específico de variação entre os acompanhamentos para definir uma mudança com importância clínica no desfecho de um paciente (EN: 2 pontos; QIRM: 5 pontos; EQ-5D: 0,03 ponto) entre os achados antes e depois da cirurgia, é usada como definição de recuperação. ${ }^{20-22}$

\section{Tamanho da Amostra}

Como este estudo é uma coorte retrospectiva, o tamanho da amostra é limitado ao número de pacientes elegíveis submetidos ao tratamento completo entre junho de 2011 e janeiro de 2014.

A partir de um estudo anterior ${ }^{21}$, estimamos o escore médio de dor em 5,8, com desvio padrão (DP) de 2,0. Considerando o objetivo principal de comparação do nível de dor em grupos submetidos a diferentes tratamentos e assumindo que o DP da escala de dor neste estudo é semelhante ao observado por Childs et al., ${ }^{21}$ a amostra é suficiente para atingir um poder superior a $95 \%$ para a detecção de uma diferença de 1 ponto na escala de dor ao usar o delineamento de medidas repetidas com estrutura de correlação, assumindo que a correlação entre as medidas de um mesmo paciente ao longo do tempo é de 0,5 , e o nível de significância, de $5 \%$. Os cálculos foram realizados usando-se o pacote de 
Tabela 1 Características demográficas e clínicas do estudo em valores expressos como média (desvio padrão ou porcentagem)

\begin{tabular}{|c|c|c|c|}
\hline & Fusão espinhal $(n=22)$ & $\begin{array}{l}\text { Descompressão lombar } \\
(n=49)\end{array}$ & $\begin{array}{l}\text { Total } \\
(n=71)\end{array}$ \\
\hline Sexo masculino* & $11(50 \%)$ & $27(55,1 \%)$ & $38(53,5 \%)$ \\
\hline Idade, anos* & $59,14(15,65)$ & $44,51(15,15)$ & $62,47(8,66)$ \\
\hline Índice de massa corporal, $\mathrm{kg} / \mathrm{m}^{2^{*}}$ & $28,96(2,97)$ & $27,19(4,91)$ & $27,7(4,4)$ \\
\hline Tabagismo, sim * & $5(22,72 \%)$ & $9(18,36 \%)$ & $14(19,7 \%)$ \\
\hline \multicolumn{4}{|l|}{ Comorbidades* } \\
\hline Hipertensão, sim & $5(22,72 \%)$ & $12(24,48 \%)$ & $17(23,9 \%)$ \\
\hline Diabetes, sim & $2(9,09 \%)$ & $2(4,08 \%)$ & $4(5,6 \%)$ \\
\hline Histórico de câncer, sim & $1(4,54 \%)$ & $1(2,04 \%)$ & $2(2,8 \%)$ \\
\hline \multicolumn{4}{|l|}{ Diagnóstico } \\
\hline Radiculopatia/Hérnia de disco/Protrusão de disco & $7(31,81 \%)$ & $37(75,51 \%)$ & $44(62,0 \%)$ \\
\hline Estenose do canal lombar & $4(18,18 \%)$ & $3(6,12 \%)$ & $7(9,9 \%)$ \\
\hline Dor lombar mecânica & $3(13,63 \%)$ & $2(4,08 \%)$ & $5(7,0 \%)$ \\
\hline Espondilólise/Espondilolistese & $3(13,63 \%)$ & $1(2,04 \%)$ & $4(5,6 \%)$ \\
\hline Doença do disco intervertebral & $3(13,63 \%)$ & $2(4,08 \%)$ & $5(7,0 \%)$ \\
\hline Desconhecido & $2(9,09 \%)$ & $4(8,16 \%)$ & $6(8,5 \%)$ \\
\hline Reoperações & 0 & $3(4 \%)$ & $3(4 \%)$ \\
\hline
\end{tabular}

Nota: ${ }^{*} p>0,05$ em todas as características analisadas.

programas Power Analysis and Sample Size 14 (PASS 14, NCSS, LLC, Kaysville, UT, Estados Unidos).

\section{Análise Estatística}

Os dados foram descritos como frequências absolutas e relativas para variáveis categóricas e DPs ou medianas e quartis, bem como valores mínimos e máximos, para variáveis numéricas. Modelos mistos generalizados foram ajustados para investigar diferenças nas pontuações entre os acompanhamentos para considerar a dependência entre as avaliações de um mesmo paciente. As diferenças relativas aos achados pré-cirúrgicos entre os grupos foram analisadas pelo teste $t$ não pareado, e as diferenças nas pontuações do instrumento entre os momentos de avaliação foram analisadas com modelos mistos generalizados. As diferenças foram detectadas em testes de comparações múltiplas corrigidos pelo método sequencial de Bonferroni, avaliando os efeitos antes e depois da cirurgia, bem como a interação entre os momentos de análise. Os resultados foram apresentados como valores médios ajustados pelos modelos e intervalos de confiança de $95 \%$. As análises foram realizadas no programa Statistical Package for the Social Sciences (SPSS, IBM Corp., Armonk, NY, Estados Unidos), considerando-se um nível de significância de $5 \%$.

Este estudo foi aprovado pelo Comitê de Ética em Pesquisa local (CAAE: 59736016.0.0000.0071). Todos os participantes assinaram um termo de consentimento em que concordaram em participar do programa de coluna.

\section{Resultados}

A amostra do estudo foi composta por 71 pacientes com idade igual ou superior a 18 anos e indicação de cirurgia (com encaminhamento por profissional de saúde) para tratamento de doenças degenerativas da coluna lombar. Todos participaram do programa de segunda opinião em cirurgias de coluna, realizado em hospital terciário com acesso à saúde suplementar, e foram submetidos ao tratamento cirúrgico e acompanhados por 36 meses. As características demográficas e clínicas basais dos pacientes são mostradas na - Tabela 1 de acordo com 0 procedimento cirúrgico. Ao comparar os dois tipos de procedimentos cirúrgicos, observamos diferenças significativas nas características demográficas e clínicas e nos diagnósticos dos pacientes de cada grupo $(p>0,05)$. Houve maior prevalência de descompressão lombar (69\%); além disso, os grupos apresentaram diferenças estatísticas ( $p>0,05)$ em todas as características analisadas. Em relação às taxas de reoperação, apenas a descompressão lombar apresentou uma taxa cirúrgica de $4 \%(n=3)$ para hérnia de disco lombar.

Quanto às comparações de achados antes e depois das intervenções cirúrgicas, a - Tabela 2 mostra que a DMCI foi alcançada em todos os desfechos de qualidade de vida, dor e função nos dois períodos de acompanhamento em relação aos escores basais ( $p<0,001$; - Tabela 2 ).

No entanto, ao analisar os momentos pós-operatórios sem considerar o procedimento cirúrgico, não foram observadas diferenças ou um valor específico de variação entre 12 e 36 meses de acompanhamento para definir uma mudança clinicamente significativa nos desfechos de qualidade de vida, dor, e função (-Tabela 2 ).

\section{Discussão}

As intervenções cirúrgicas são frequentemente consideradas uma opção para o tratamento da lombalgia, apesar das divergências quanto à escolha da técnica. ${ }^{2,3,5}$ Portanto, o objetivo deste estudo foi comparar os desfechos em longo prazo de qualidade de vida, dor e função após a descompressão lombar ou a fusão espinhal em pacientes com patologias degenerativas 
Tabela 2 Valores ajustados médios e intervalos de confiança de 95\% das pontuações dos instrumentos de qualidade de vida, dor e função antes da cirurgia e durante o acompanhamento de acordo com a intervenção cirúrgica.

\begin{tabular}{|c|c|c|c|c|}
\hline Instrumentos & $\begin{array}{l}\text { Antes da cirurgia } \\
(\mathrm{N}=71)\end{array}$ & $\begin{array}{l}12 \text { meses após a } \\
\text { cirurgia } \\
(\mathrm{N}=68)\end{array}$ & $\begin{array}{l}36 \text { meses após } \\
\text { a cirurgia } \\
(\mathrm{N}=67)\end{array}$ & valor de $p$ \\
\hline \multicolumn{5}{|l|}{ EuroQoL-5D } \\
\hline Fusão espinhal & $0,39(0,28-0,49)$ & $0,72(0,61-0,82)$ & $0,69(0,58-0,80)$ & \\
\hline Descompressão lombar & $0,37(0,30-0,44)$ & $0,77(0,70-0,84)$ & $0,83(0,76-0,91)$ & \\
\hline Total & $0,38(0,32-0,44)$ & $0,75(0,69-0,81)$ & $0,79(0,73-0,85)$ & $<0,001$ \\
\hline Diferença mínima clinicamente importante & - & 0,37 & 0,41 & $0,356^{\dagger}$ \\
\hline \multicolumn{5}{|l|}{ Escala Numérica de Classificação de Dor } \\
\hline Fusão espinhal & $7,8(6,8-9,0)$ & $4,5(3,7-5,4)$ & $4,0(3,2-5,1)$ & \\
\hline Descompressão lombar & $8,2(7,5-9,0)$ & $4,5(3,9-5,1)$ & $4,4(3,8-5,1)$ & \\
\hline Total & $8,1(7,5-8,7)$ & $4,5(4,0-5,0)$ & $4,3(3,8-4,8)$ & $<0,001$ \\
\hline Diferença mínima clinicamente importante & - & 3,6 & 3,8 & $0,559^{\dagger}$ \\
\hline \multicolumn{5}{|l|}{ Questionário de Incapacidade de Roland Morris } \\
\hline Fusão espinhal & $14,8(11,8-18,5)$ & $6,4(4,5-9,0)$ & $5,9(4,0-8,6)$ & \\
\hline Descompressão lombar & $16,4(14,2-18,9)$ & $5,8(4,5-7,4)$ & $4,8(3,7-6,3)$ & \\
\hline Total & $15,9(14,1-17,9)$ & $6,0(4,9-7,3)$ & $5,1(4,1-6,4)$ & $<0,001$ \\
\hline Diferença mínima clinicamente importante & & 9,9 & 10,8 & $0,200^{\dagger}$ \\
\hline
\end{tabular}

Nota: †Valores de $p$ corrigidos pelo método sequencial de Bonferroni.

da coluna vertebral que participaram de um programa de segunda opinião para cirurgias da coluna vertebral.

Nossos resultados apoiam as evidências atuais sobre melhoras clinicamente significativas após a cirurgia, ${ }^{23}$ e destacam que as pontuações não diferiram entre os pacientes submetidos à fusão espinhal ou descompressão lombar, mas variaram entre os momentos ( $p<0,001)$, uma vez que o escore pré-operatório médio foi superior aos valores médios $(p<0,001)$ dos acompanhamentos pós-operatórios aos 12 e 36 meses.

Por outro lado, já se sabe que os desfechos positivos, as baixas taxas de complicações e reoperações, e a boa relação custobenefício são associados à escolha de pacientes para determinadas técnicas cirúrgicas, muitas vezes com base no perfil individual e nas comorbidades específicas, além de idade e índice de massa corporal (IMC). ${ }^{24}$ Da mesma forma, evidências recentes sugerem que a fusão não oferece mais benefícios em comparação com a cirurgia de descompressão tradicional. ${ }^{25}$ Além disso, as evidências mostram que a incidência de reoperações de coluna é de $5 \%$ a $16 \%$, dependendo dos fatores de risco; ${ }^{26,27}$ assim, este estudo apresentou desfechos positivos e taxa mínima de recidiva de hérnia de disco lombar (4\%), observada apenas no grupo submetido à descompressão lombar.

Ademais, os pacientes deste estudo fazem parte de um programa de segunda opinião que pode ser considerado uma alternativa à abordagem de tomada de decisão compartilhada para validar ou não a realização de uma cirurgia para dor nas costas. $^{28}$ Esta interação entre o paciente e o profissional de saúde comprovadamente melhora a adesão do paciente e reduz o tempo de cicatrização e os déficits funcionais. ${ }^{29}$

Dados descritivos e administrativos associados aos desfechos foram apresentados por pesquisadores na tentativa de relatar taxas de procedimentos com base populacional, resultados de segurança cirúrgica, e custos do diagnóstico e tratamento de doenças relacionadas à dor nas costas. ${ }^{15,30}$ Nossos resultados contribuem para o corpo de evidências atual, e demonstram que pacientes com patologias espinhais degenerativas apresentam resultados clinicamente significativos de dor, função e qualidade de vida em longo prazo, independentemente da intervenção cirúrgica.

Além disso, devido à heterogeneidade de sintomas e patologia, é bom afirmar que não há critérios padronizados para encaminhar pacientes a grupos clinicamente significativos com base na indicação cirúrgica. ${ }^{31}$ Este estudo aponta uma maior taxa de descompressão lombar em um programa de cirurgia de coluna, mas isso pode estar relacionado a vários motivos, como o uso arbitrário de técnicas operatórias pelos cirurgiões, manejo hospitalar, e abordagens compartilhadas de tomada de decisão.

O tratamento cirúrgico é bastante controverso, e muitas técnicas foram desenvolvidas e realizadas em todo o mundo. A fusão espinhal e a descompressão lombar são as 2 cirurgias lombares mais realizadas, e têm sido associadas a desfechos ruins em $20 \%$ a $40 \%$ dos pacientes. ${ }^{30,31}$ Em nosso estudo, observamos diversos diagnósticos com a mesma indicação cirúrgica, o que leva à necessidade de mais estudos para aprimorar os critérios de indicação, reduzir as taxas de reoperação, e melhorar a qualidade do tratamento.

\section{Conclusão}

Em conclusão, pacientes com patologias degenerativas da coluna vertebral apresentam desfechos de dor, função e qualidade de vida em longo prazo que são clinicamente 
significativos, independentemente da intervenção cirúrgica. No entanto, novos estudos devem ser realizados para melhorar a compreensão dos fatores que contribuem para esses desfechos e do impacto econômico associado a cada intervenção.

\section{Contribuições dos Autores}

Todos os autores conceberam o estudo. IOO escolheu o delineamento experimental, e ML, EA e MF contribuíram para a versão final deste manuscrito. IOO e EA participaram da análise estatística. Todos os autores contribuíram para o refinamento do estudo e aprovaram o manuscrito final.

\section{Suporte Financeiro}

Não houve suporte financeiro de fontes públicas, comerciais, ou sem fins lucrativos.

\section{Conflito de Interesses}

Os autores declaram não haver conflito de interesse.

\section{Agradecimentos}

Os autores gostariam de agradecer ao grupo de consultoria em estatística do Escritório de Apoio ao Pesquisador do Hospital Israelita Albert Einstein pelo apoio técnico, e à toda a equipe do Projeto Coluna do Hospital Israelita Albert Einstein pelo apoio no cuidado com os pacientes.

\section{Referências}

1 Gakidou E, Afshin A, Abajobir AA, et al. GBD 2016 Risk Factors Collaborators. Global, regional, and national comparative risk assessment of 84 behavioural, environmental and occupational, and metabolic risks or clusters of risks, 1990-2016: a systematic analysis for the Global Burden of Disease Study 2016. Lancet 2017;390(10100):1345-1422

2 Kaiser MG, Eck JC, Groff MW, et al. Guideline update for the performance of fusion procedures for degenerative disease of the lumbar spine. Part 1: introduction and methodology. J Neurosurg Spine 2014;21(01):2-6

3 Jain N, Acharya S, Adsul NM, et al. Lumbar Canal Stenosis: A Prospective Clinicoradiologic Analysis. J Neurol Surg A Cent Eur Neurosurg 2020;81(05):387-391

4 Verbiest H. A radicular syndrome from developmental narrowing of the lumbar vertebral canal. J Bone Joint Surg Br 1954;36 (02):230-237

5 Zaina F, Tomkins-Lane C, Carragee E, Negrini S. Surgical versus non-surgical treatment for lumbar spinal stenosis. Cochrane Database Syst Rev 2016;2016(01):CD010264

6 Siebert E, Prüss H, Klingebiel R, Failli V, Einhäupl KM, Schwab JM. Lumbar spinal stenosis: syndrome, diagnostics and treatment. Nat Rev Neurol 2009;5(07):392-403

7 Barrick WT, Schofferman JA, Reynolds JB, et al. Anterior lumbar fusion improves discogenic pain at levels of prior posterolateral fusion. Spine (Phila Pa 1976) 2000;25(07):853-857

8 Phillips FM, Slosar PJ, Youssef JA, Andersson G, Papatheofanis F. Lumbar spine fusion for chronic low back pain due to degenerative disc disease: a systematic review. Spine (Phila Pa 1976) 2013; 38(07):E409-E422

9 Fritzell P, Hägg O, Wessberg P, Nordwall ASwedish Lumbar Spine Study Group. Chronic low back pain and fusion: a comparison of three surgical techniques: a prospective multicenter randomized study from the Swedish lumbar spine study group. Spine (Phila Pa 1976) 2002;27(11):1131-1141
10 Arai Y,Takahashi M, Kurosawa H, Shitoto K. Comparative study of iliac bone graft and carbon cage with local bone graft in posterior lumbar interbody fusion. J Orthop Surg (Hong Kong) 2002;10(01):1-7

11 Gibson JNA, Waddell G. Surgery for degenerative lumbar spondylosis: updated Cochrane Review. Spine 2005;30(20):2312-2320

12 Kim KT, Lee SH, Lee YH, Bae SC, Suk KS. Clinical outcomes of 3 fusion methods through the posterior approach in the lumbar spine. Spine (Phila Pa 1976) 2006;31(12):1351-1357

13 Miyauchi A. Decompression Alone is Effective in Lumbar Spinal Stenosis with Degenerative Spondylolisthesis. Spine J 2012;12 (09):S92-S93

14 Miller JW, Sasso RC. Lumbar extraforaminal decompression: A technical note and retrospective study looking at potential complications as an outpatient procedure. SAS J 2011;5(01):4-8

15 Deyo RA, Mirza SK, Martin BI. Error in trends, major medical complications, and charges associated with surgery for lumbar spinal stenosis in older adults. JAMA 2011;306(10):1088

16 Costa LO, Maher CG, Latimer J, et al. Clinimetric testing of three selfreport outcome measures for low back pain patients in Brazil: which one is the best? Spine (Phila Pa 1976) 2008;33(22):2459-2463

17 EuroQol Group. EuroQol-a new facility for the measurement of health-related quality of life. Health Policy 1990;16(03):199-208

18 Nusbaum L, Natour J, Ferraz MB, Goldenberg J. Translation, adaptation and validation of the Roland-Morris questionnaireBrazil Roland-Morris. Braz J Med Biol Res 2001;34(02):203-210

19 Grotle M, Brox JI, Vøllestad NK. Concurrent comparison of responsiveness in pain and functional status measurements used for patients with low back pain. Spine (Phila Pa 1976) 2004;29(21):E492-E501

20 Soer R, Reneman MF, Speijer BL, Coppes MH, Vroomen PC. Clinimetric properties of the EuroQol-5D in patients with chronic low back pain. Spine J 2012;12(11):1035-1039

21 Childs JD, Piva SR, Fritz JM. Responsiveness of the numeric pain rating scale in patients with low back pain. Spine (Phila Pa 1976) 2005;30(11):1331-1334

22 Stratford PW, Binkley J, Solomon P, Finch E, Gill C, Moreland J. Defining the minimum level of detectable change for the RolandMorris questionnaire. Phys Ther 1996;76(04):359-365

23 Alentado VJ, Caldwell S, Gould HP, Steinmetz MP, Benzel EC, Mroz TE. Independent predictors of a clinically significant improvement after lumbar fusion surgery. Spine J 2017;17(02):236-243

24 Soegaard R, Christensen FB, Christiansen T, Bünger C. Costs and effects in lumbar spinal fusion. A follow-up study in 136 consecutive patients with chronic low back pain. Eur Spine J 2007;16 (05):657-668

25 Machado GC, Maher CG, Ferreira PH, et al. Trends, Complications, and Costs for Hospital Admission and Surgery for Lumbar Spinal Stenosis. Spine (Phila Pa 1976) 2017;42(22):1737-1743

26 Camino Willhuber G, Kido G, Mereles M, et al. Factors associated with lumbar disc hernia recurrence after microdiscectomy. Rev Esp Cir Ortop Traumatol 2017;61(06):397-403

27 Piper K, DeAndrea-Lazarus I, Algattas H, et al. Risk Factors Associated with Readmission and Reoperation in Patients Undergoing Spine Surgery. World Neurosurg 2018;110:e627-e635

28 Oliveira IO, Lenza M, Vasconcelos RA, Antonioli E, Cendoroglo Neto M, Ferretti M. Second opinion programs in spine surgeries: an attempt to reduce unnecessary care for low back pain patients. Braz J Phys Ther 2019;23(01):1-2

29 Gaudin D, Krafcik BM, Mansour TR, Alnemari A. Considerations in Spinal Fusion Surgery for Chronic Lumbar Pain: Psychosocial Factors, Rating Scales, and Perioperative Patient Education-A Review of the Literature. World Neurosurg 2017;98:21-27

30 Martin BI, Deyo RA, Mirza SK, et al. Expenditures and health status among adults with back and neck problems. JAMA 2008;299(06): 656-664

31 Martin BI, Mirza SK, Franklin GM, Lurie JD, MacKenzie TA, Deyo RA. Hospital and surgeon variation in complications and repeat surgery following incident lumbar fusion for common degenerative diagnoses. Health Serv Res 2013;48(01):1-25 